\title{
Bioelectronics as a Possible Therapeutic Modality Targeting Sphincteric Dysfunction in Gastrointestinal Disorders
}

\author{
Larry Miller ${ }^{1}$ and Anil Vegesna ${ }^{2}$
}

${ }^{1}$ Department of Medicine, Division of Gastroenterology, North Shore-Long Island Jewish Health System, Manhasset, New York, United States of America; and ${ }^{2}$ Department of Medicine, Division of Gastroenterology, The Feinstein Institute for Medical Research, Manhasset, New York, United States of America

\begin{abstract}
Sphincters in the gastrointestinal tract control the movement of luminal contents in the forward direction and prevent their movement in the backward direction. Defective sphincters are responsible for many of the disorders in the gastrointestinal tract. The defects might be due to abnormally loose lower esophageal or anal sphincters, with the result being diseases such as gastroesophageal reflux disease and fecal incontinence, respectively. In some cases the lower esophageal or anal sphincter might be too tight and thus prevent the forward movement of gastrointestinal luminal contents, with the result being diseases such as achalasia or functional rectosigmoid obstruction, respectively. There are multiple causes that might lead to the disruption of the normal function of these sphincters. The probable causes include damage to the nerves supplying the sphincter or to the sphincter itself either owing to trauma or owing to infection or from congenital diseases. The majority of the causes responsible for sphincteric dysfunction are still unknown. Because the majority of the causes of sphincteric dysfunction are unknown, there are very few effective pharmacologic treatments for many of these conditions. For example, all the current treatments for gastroesophageal reflux disease are aimed at treating the symptoms by decreasing the stomach acid. These treatments do little to prevent the actual event of reflux. Bioelectronic medicine may play a major role in treating these conditions by directly manipulating either the sphincter or the nerves that supply the sphincters. Because this is a targeted approach, one can expect to have better efficiency and fewer side effects than traditional pharmacological treatments.
\end{abstract}

Online address: www.bioelecmed.org

doi: 10.15424/bioelectronmed.2014.00005

\section{INTRODUCTION}

A sphincter in the gastrointestinal (GI) tract is a ring-like muscle that normally maintains constriction of a body passage or orifice and relaxes as required by normal physiological functioning. Sphincters in the gastrointestinal tract control forward passage and prevent or allow the backwards passage of solids, liquids and gases. Some of the examples of GI sphincters are upper esophageal sphincter, lower esophageal sphincter, pyloric sphincter, sphincter of oddi, Ileocecal sphincter and anal sphincter. Defective sphincters are responsible for many of the functional disorders in the GI tract. In general sphincter disorders in the GI tract are due to the sphincter muscle being too loose, as in gastroesophageal reflux disease (GERD) or fecal incontinence, or the sphincter muscles being too tight as in achalasia in the esophagus or functional rectosigmoid obstruction in the

Address correspondence to Larry Miller, Department of Medicine, Division of Gastroenterology, North Shore-Long Island Jewish Health System, Fourth Floor Levitt Pavilion, 350 Community Drive, Manhasset, NY 11030. Phone: 718-470-4691; Fax: 718-343-0128; E-mail: Imiller7@nshs.edu.

Submitted August 11, 2014; Accepted for publication September 9, 2014; Published Online (www.bioelecmed.org) December 2, 2014.

\section{The Feinstein Institute for Medical Research} Empowering Imagination. Pioneering Discovery.

anal sphincter. Many of these sphincteric dysfunctions may be targeted for future therapy by using devices that deliver electrical current to the sphincteric muscle or to the nerves innervating the muscle to either relax or to contract the sphincter in an appropriate manner. The first attempts at electrical stimulation to affect gastrointestinal motility occurred in the 1960s and 1970s. Although most of these efforts were directed toward gastric electrical stimulation to treat gastroparesis, there have been more recent attempts at electrical stimulation of the sphincter directly or to the nerves innervating the sphincter.

The following are examples of sphincter dysfunction causing functional gastrointestinal disorders, which we have studied in our laboratory. These studies describe the normal physiology and the underlying pathophysiology of GERD and the role of the ileocecal valve in small bowel bacterial overgrowth. These 


\section{Gastroesophageal junction pressures in non-GERD subjects $(n=15)$}

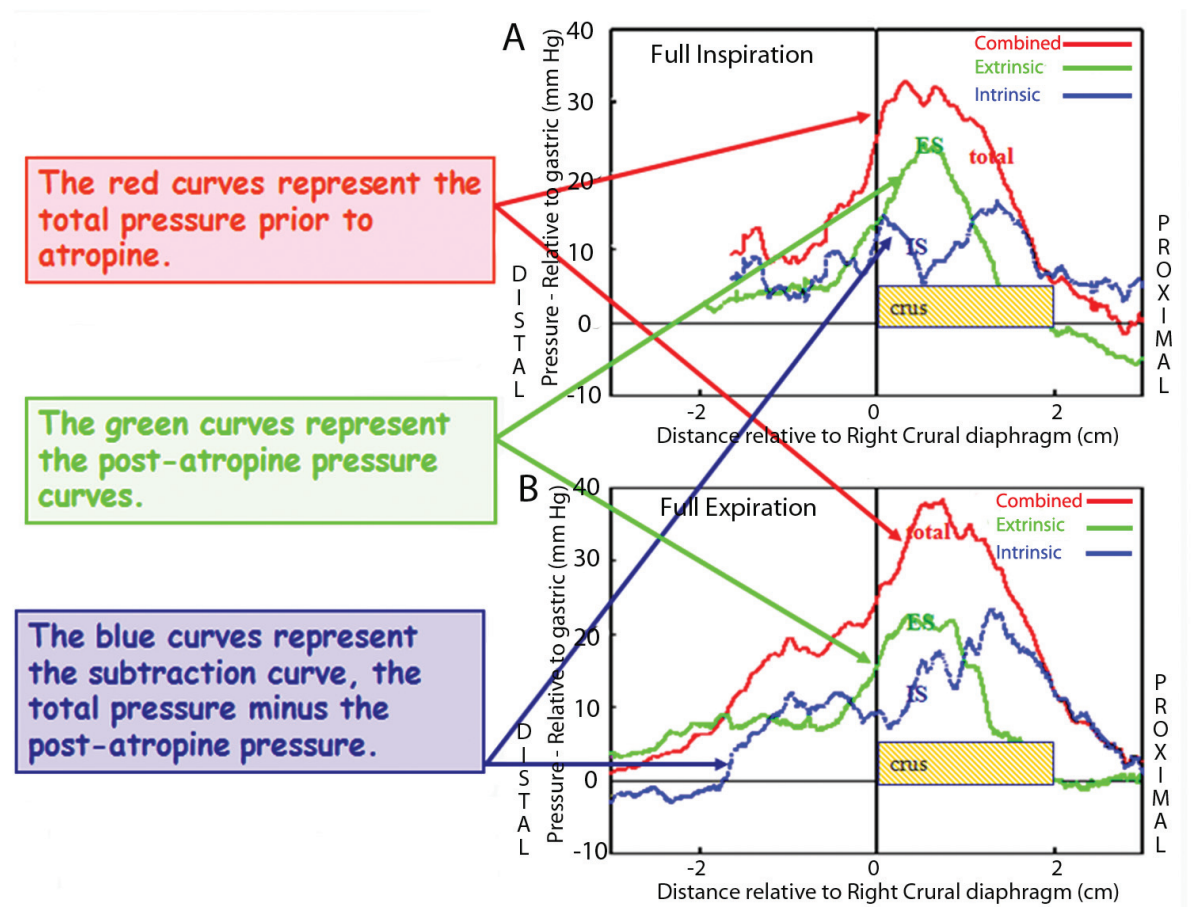

Figure 1. Extrinsic and intrinsic components for the GEJHPZ in 15 normal control subjects. Panel A represents the pressure components during full inspiration. Panel B represents the pressure components during full expiration. The $x$ axis represents the relative distance from the start of right crural diaphragm, and the $y$ axis represents the pressures relative to the gastric pressures. The red curves are preatropine pressures, reflecting the combined external and internal sphincter pressures. The green curves are the postatropine pressures (extrinsic crural sphincter pressures). The blue curves are the subtracted (preatropine minus postatropine) pressures, reflecting the muscarinic intrinsic sphincter components. The intrinsic sphincter pressure curves display two peaks in both full inspiration and full expiration. The distal peak reflects the gastric sling fiber/clasp fiber complex, whereas the proximal peak reflects a physiologic LEC within the abdominal esophagus that overlaps the crural sphincter.

identified sphincteric defects can be targeted by using bioelectronics to treat diseases.

\section{THE HIGH-PRESSURE ZONE OF THE DISTAL ESOPHAGUS AND PROXIMAL STOMACH IN GERD}

Gastroesophageal junction highpressure zone (GEJHPZ) is comprised of various components which include intrinsic smooth muscle in the wall of the lower esophagus and upper stomach and extrinsic skeletal muscle (crural diaphragm) acting as a sphincter from outside the wall of the esophagus. In several of our prior studies $(1,2)$ we have used simultaneous ultrasound and manometry with pharmacologic manipulation to study the anatomy and physiology of the various components of GEJHPZ in non-GERD subjects. Highfrequency ultrasound was colocalized with manometric pressure and this catheter assembly was pulled distal to proximal from the stomach into the thoracic esophagus. This was performed during breath holding under full inspiration and full expiration. All pressures were referenced to intragastric pressure. The inferior margin of the right crus of the diaphragm (RCd) and the initiation of the pull-through (pull-through start position) were used as axial spatial references. Pull-throughs were performed before and after intravenous administration of atropine to attenuate the pressure from the muscarinic intrinsic sphincter components (smooth muscle components). The muscarinic contribution to the intrinsic sphincter was reconstructed by subtracting the postatropine pressure profiles from the preatropine pressure profiles referenced to the crural diaphragm on the ultrasound image.

The muscarinic component of the intrinsic sphincter demonstrates two distinct peaks in both inspiration and expiration that are separated by roughly $1 \mathrm{~cm}$ during the displacement of the costal diaphragm from its inferior to its superior-most positions. The distal peak reflects the intrinsic pressure due to the gastric clasp and gastric sling muscles (GC/GS), whereas the proximal peak reflects the pressure due to the lower esophageal circular muscle sphincter (LEC) (Figure 1). By superimposing the three pressure curves (preatropine total pressures, postatropine atropine-resistant pressures, and the subtracted muscarinic pressure contribution), it was possible to separate and study each of the sphincteric components of the normal GEJHPZ individually and as an overlapping group for the first time in vivo and to determine how they move with respect to each other during respiration.

To determine the extent to which the muscarinic component of the intrinsic sphincter (subtracted curves) approximated the complete intrinsic sphincter, a second study was performed in which the crural diaphragm was pharmacologically paralyzed (the external sphincter, rather than the internal sphincters as in the previous study) to isolate directly the intrinsic sphincteric contribution to the GEJHPZ (1). Non-GERD subjects undergoing general anesthesia who were on a ventilator for nonesophageal surgery were evaluated in this study. Cisatracurium was given to the subjects to paralyze the crural diaphragm. The probe was withdrawn at a constant ve- 
locity, and ultrasound images and pressure tracings were recorded simultaneously during inspiratory pause on a ventilator. Ensemble averaging of pressure was performed and referenced to the inferior margin of the crural diaphragm, as in the previous atropine study. Comparisons were made between the intrinsic sphincter pressure profile from the cisatracurium study, and the muscarinic intrinsic sphincter pressure profile from the atropine study. Two pressure peaks in the same relative axial locations with respect to the crural diaphragm were found in both pressure profiles from both these studies (Figure 2), indicating that the muscarinic contribution to pressure with the double-peaked structure was a good approximation of the intrinsic component of the gastroesophageal sphincter.

Thus the intrinsic sphincter is composed of two components, a proximal component (LEC) that overlaps and moves with the crural sphincter, and a distal component at the junction between the gastric cardia and esophagus, formed by the GC/GS complex. These two components along with the crural diaphragm combine to make up the high-pressure zone of the distal esophagus and proximal stomach which acts as an antireflux barrier (1).

Using the same techniques, we (3), evaluated patients with GERD. The relative contributions of each of the components of the GEJHPZ in GERD patients were evaluated and compared with non-GERD subjects, to determine which abnormalities may predispose patients to reflux. In the GERD patients, the intrinsic sphincter pressure profiles displayed an absence of the distal pressure in both inspiration (Figure 3 ) and expiration, indicating an absence of muscarinic tone in the GC/GS complex. In addition, the proximal pressure in patients with GERD reflecting the LEC pressure was present in the same axial location relative to the crural diaphragm as in the non-GERD subjects, but was attenuated by approximately $50 \%$ in magnitude.

Comparision of Gastroesophageal junction smooth muscle pressures in non-GERD subjects
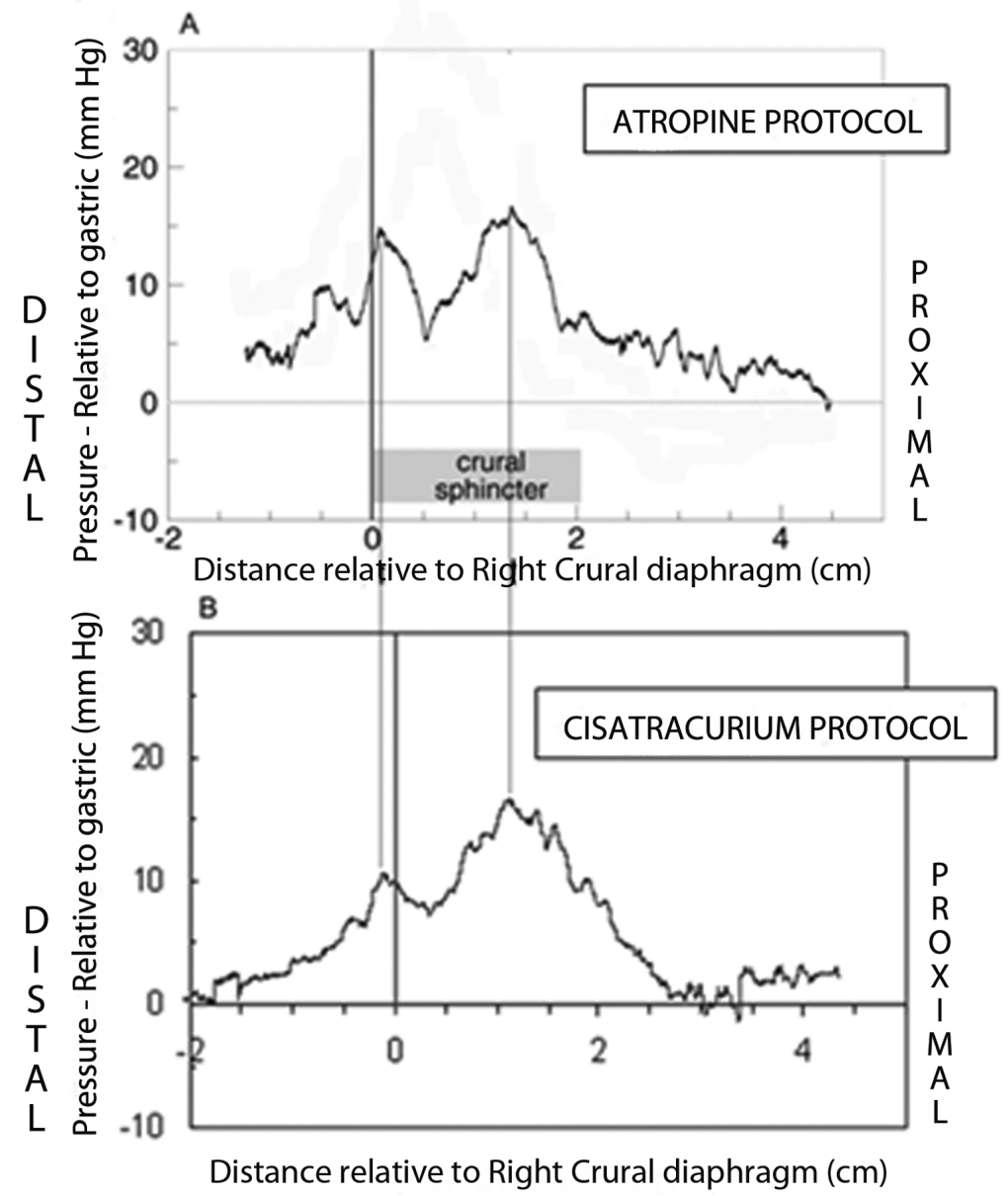

Figure 2. Comparison between smooth muscle pressures obtained from two different protocols. In panel A, the atropine-resistant pressures are subtracted from full pressure profiles, while in panel B the full smooth-muscle pressure distributions were measured directly after pharmacologically attenuating the skeletal crural muscle with cisatracurium. The $x$ axis represents the relative distance from the start of right crural diaphragm, and the $y$ axis represents the pressures relative to the gastric pressures. The pressures in panel A were obtained under full inspiration, while the pressures in panel B were obtained during maximal lung inflation and inspiratory pause. Both curves are ensemble averages after referencing to the RCd. The curve in panel B has been shifted by $2 \mathrm{~mm}$ to demonstrate the alignment with the proximal and distal peaks in panel $A$.

\section{THE MUSCULARIS MUCOSA AND ITS ROLE IN GERD}

In another study, we evaluated the movement of the mucosa/muscularismucosa/submucosa (MMS) at the GEJHPZ in non-GERD subjects and in patients with GERD (4). The GEJHPZ was evaluated during 5-mL swallows by use of two different methods. The first method used high-resolution endolumi- nal ultrasound and manometry while the second method used transabdominal ultrasound. By using endoluminal ultrasound and manometry, the changes in luminal pressures and changes in the cross-sectional area of esophageal wall layers were measured. By using transabdominal ultrasound, MMS movement at the GEJHPZ was analyzed. By using endoluminal ultrasound, it was found 


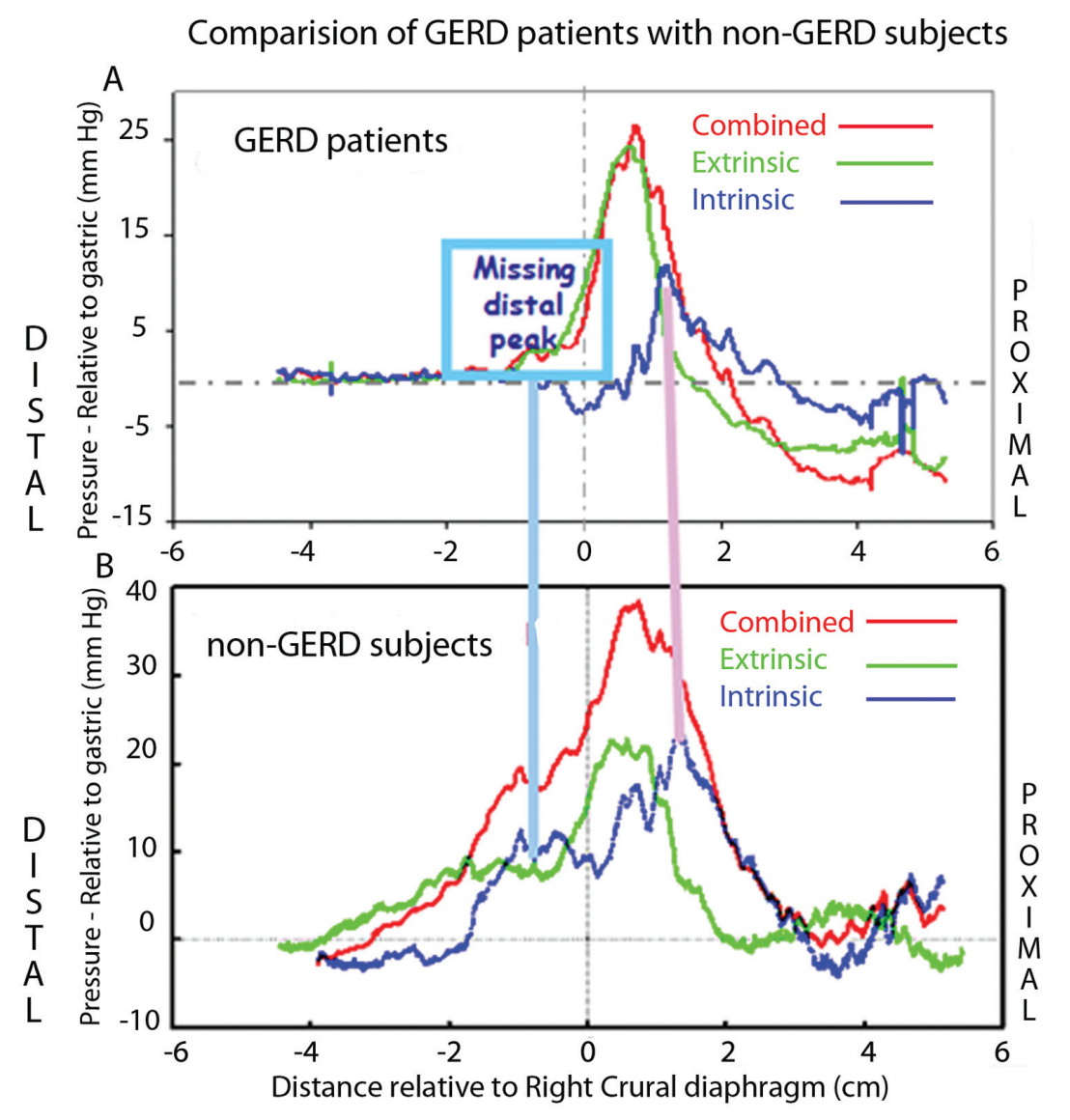

Figure 3. Extrinsic and intrinsic components for the GEJHPZ in seven GERD patients compared with that of non-GERD subjects. Panel A shows the ensemble averaged pressure curve in full expiration from seven GERD patients. Panel B shows the ensemble averaged pressure curve in full expiration. The $x$ axis represents the relative distance from the start of right crural diaphragm, and the $y$ axis represents the pressures relative to the gastric pressures. The red curve is the preatropine pressure profile, the green curve is the postatropine pressure profile and the blue curve is the subtraction profile (red curve minus green curve). Note that in the subtraction curve (blue), the proximal intrinsic muscarinic pressure peak is present in the GERD patients and is in the same axial position relative to the RCd as in the normal volunteers as demonstrated by the rose-colored vertical line on the right. However, the magnitude of this pressure peak is less in the GERD patients. In GERD patients, the distal intrinsic muscarinic peak is absent, as demonstrated by the light turquoise-colored line and box on the left (missing distal peak).

that, in the non-GERD subjects, the gastric MMS moved rostrally into the distal esophagus to form a functional barrier to reflux of gastric contents at the time when the pressure within the GEJHPZ is minimal due to relaxation of these muscle groups during swallowing. However, in GERD patients, the gastric MMS did not move rostrally into the distal esophagus. The maximum change in the crosssectional area of gastroesophageal MMS in non-GERD subjects and in GERD patients was $289 \%$ and $183 \%$, respectively (Figure 4). The independent movement of the gastric MMS in GERD subjects was significantly less than in non-GERD subjects. In GERD patients, movement of the MMS does not occur, thus there is no anatomic or physiologic barrier to reflux of gastric contents during swallowing. We concluded that in non-GERD subjects, the gastric MMS moves rostrally into the distal esophagus during swallowing to act as an antireflux barrier, by causing a functional obstruction to reflux of gastric contents during relaxation of the GEJHPZ. This rostral movement of gastric MMS during swallowing is absent in GERD patients. Therefore, if the pressure in the abdominal cavity exceeds the pressure in the thoracic cavity there will be free reflux of gastric contents in the GERD patients, as was demonstrated on transabdominal ultrasound.

\section{THE ILEOCECAL VALVE AND ITS ROLE IN SMALL BOWEL BACTERIAL OVERGROWTH}

The ileocecal valve (ICV) is a physiological sphincter which lies at the junction of the small intestine (ileum) and the large intestine (cecum) (5). It normally acts to prevent reflux of fecal contents into the small intestine from the colon. We performed a study to evaluate whether patients with a defective ICV/cecal distension reflex have small bowel bacterial overgrowth (6). By use of a colonoscope under minimal sedation with midazolam and fentanyl, the ICV was intubated and the colonoscope was placed within the terminal ileum (TI). A manometry catheter with four pressure channels, spaced $1 \mathrm{~cm}$ apart, was passed through the biopsy channel of the colonoscope into the TI. The colonoscope was slowly withdrawn from the TI while the manometry catheter was advanced. The catheter was placed across the ICV so that at least one pressure port was within the TI, ICV and the cecum respectively. Pressures were continuously measured during air insufflation into the cecum, under direct endoscopic visualization, in 19 volunteers. Lactulose breath test was performed in all subjects one month after the colonoscopy, to identify small bowel bacterial overgrowth. The patients were grouped based on the breath test results as either positive or negative lactulose breath test. The results of the pressures within the ICV during air insufflations between the two groups were compared. The average peak ICV pressure during air insufflation into the cecum was significantly higher in sub- 
Changes in mucosal cross sectional area at GEJHPZ during swallowing

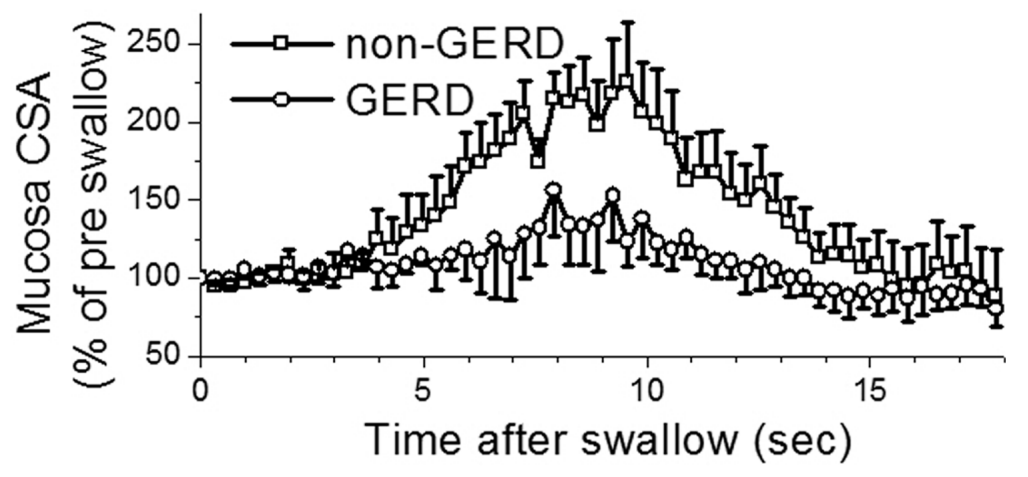

Figure 4. Changes in mucosal cross sectional area at gastroesophageal junction during swallowing. This graph shows the changes in the cross sectional area of the mucosa during swallowing in non-GERD subjects and in patients with GERD. The cross-sectional areas were measured by using endoluminal ultrasound placed in the gastroesophageal junction during swallowing. The $x$ axis represents the time from the initiation of swallow, and the $y$ axis represents the cross-sectional areas of the mucosa relative to baseline crosssectional area at the beginning of swallow. The graph shows that there is less change in the cross-sectional area of the gastric mucosa in GERD patients when compared with non-GERD subjects.

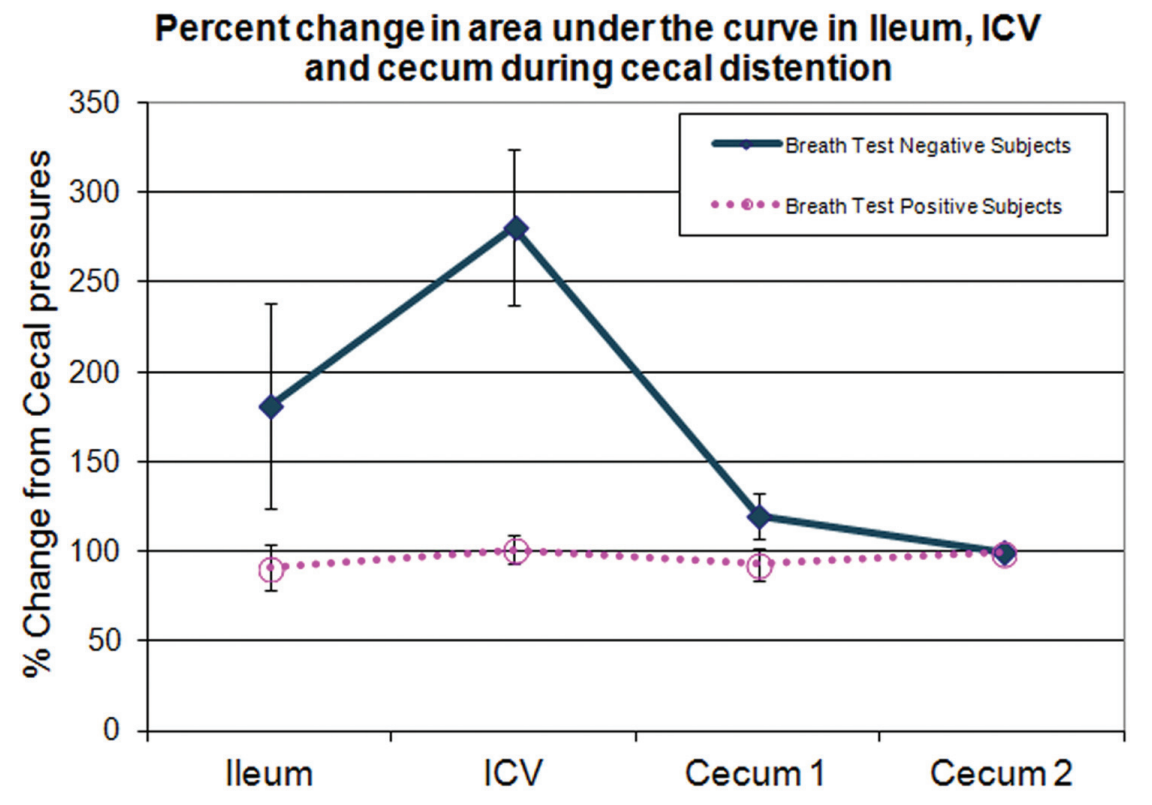

Figure 5. Percent change of the area under the pressure curve in ileum, ICV and cecum during air insufflation into the cecum in normal subjects (negative lactulose breath tests) and in subjects with small bowel bacterial over growth (positive lactulose breath tests). The $x$ axis shows the respective locations, and the $y$ axis represents the percent change in pressure with respect to cecal pressures. Note that in subjects with small bowel bacterial overgrowth there is a common cavity effect during pressurization of the cecum representing the lack of ICV/cecal distention reflex. jects with negative lactulose breath tests than in subjects with positive lactulose breath tests $(49.33 \pm 7.99 \mathrm{mmHg}$ versus $16.40 \pm 2.14 \mathrm{mmHg}, P<0.05)$. The average percent difference of the area under the pressure curve in the ICV from cecum was significantly higher in subjects with negative lactulose breath tests than in subjects with positive lactulose breath tests $(P<0.05)$ (Figure 5$)$. Individuals with positive lactulose breath tests demonstrated symptom scores which were significantly higher for not being able to finish a normal-sized meal, feeling excessively full after meals, bloating and loss of appetite. The results suggest that compared with normal subjects, subjects with small bowel bacterial overgrowth have a defective ICV/cecal distension reflex.

\section{FUTURE THERAPY FOR FUNCTIONAL GASTROINTESTINAL DISORDERS}

We are in the process of developing novel and innovative therapies to treat GI disorders targeting defective sphincteric mechanisms. GI functional disorders are some of the most common diseases worldwide. We have discovered that many of the underlying causes of functional GI disorders involve dysfunction of various sphincters. In a recent article by Rodriguez et al. (7) electrical stimulation therapy of the lower esophageal sphincter was successful in treating GERD. The final results of the trial demonstrated a significant and sustained improvement in GERD symptoms, esophageal $\mathrm{pH}$, and a reduction in PPI usage without any side effects after electrical stimulation of the LES. In another article by Hofmann et al. (8), in a canine model they evaluated the motor activity and pressure in the ileum, ICV and the colon. They found that the resting pressure at the ICV was $12.7 \pm 0.4 \mathrm{cmH}_{2} \mathrm{O}$. Electric stimulation of small mesenteric nerves to the ICV increased pressure in the ICV to $35.0 \pm 4.1 \mathrm{cmH}_{2} \mathrm{O}$. We believe that electrical stimulation of gastrointestinal sphincters holds great promise in the future treatment of functional disorders of the gastrointestinal tract. 


\section{ACKNOWLEDGMENTS}

These studies were supported by a grant from National Institute of Health (RO1 DK079954).

\section{DISCLOSURE}

The authors declare that they have no competing interests as defined by Bioelectronic Medicine, or other interests that might be perceived to influence the results and discussion reported in this paper.

\section{REFERENCES}

1. Brasseur JG, et al. (2007) Pharmacological dissection of the human gastro-oesophageal segment into three sphincteric components. J. Physiol. (Lond). 580:961-75.

2. McCray WH Jr., Chung C, Parkman HP, Miller LS. (2000) Use of simultaneous high-resolution endoluminal sonography (HRES) and manometry to characterize high pressure zone of distal esophagus. Dig. Dis. Sci. 45:1660-6.

3. Miller L, et al. (2009) A missing sphincteric component of the gastro-oesophageal junction in patients with GORD. Neurogastroenterol. Motil. 21:813-e52.

4. Vegesna AK, et al. (2014) Defective mucosal movement at the gastroesophageal junction in patients with gastroesophageal reflux disease. Dig. Dis. Sci. 59:1870-7.

5. Pollard MF, Thompson-Fawcett MW, Stringer MD. (2012) The human ileocaecal junction: anatomical evidence of a sphincter. Surg. Radiol. Anat. 34:21-9.

6. Miller LS, et al. (2012) Ileocecal valve dysfunction in small intestinal bacterial overgrowth: a pilot study. World J. Gastroenterol. 18:6801-8.

7. Rodriguez L, et al. (2013) Electrical stimulation therapy of the lower esophageal sphincter is successful in treating GERD: final results of openlabel prospective trial. Surg. Endosc. 27:1083-92.

8. Hofmann R, et al. (1993) Stimulated pressure response of the ileocolonic junctional zone and its use as a continence mechanism in a canine model. Urol. Res. 21:333-7.

Cite this article as: Miller L, Vegesna A. (2014) Bioelectronics as a possible therapeutic modality targeting sphincteric dysfunction in gastrointestinal disorders. Bioelectron. Med. 1:19-24. 